\title{
Theoretical analysis of the impact of belt speed on indentation rolling resistance
}

\author{
Lech Gładysiewicz ${ }^{1}$ and Martyna Konieczna ${ }^{1, *}$ \\ ${ }^{1}$ Wroclaw University of Science and Technology, Faculty of Geoengineering, Mining and Geology, 27 Wyb. Wyspianskiego St., \\ 50-370 Wroclaw, Poland
}

\begin{abstract}
Belt conveyors, having high reliability and efficiency, are the main means of continuous transportation both in underground and in open-cast mining. Despite their numerous advantages, however, belt conveyors are expensive in maintenance. Therefore, improvements in the field of belt conveyor transportation largely focus on methods for lowering their power consumption. The energy consumption level of a belt conveyor depends on the motion resistances which occur during its operation. In the case of conveyors having lengths greater than $80 \mathrm{~m}$, main resistances are the dominant component of motion resistances, and up to $60 \%$ of main resistances may be accounted for by the rolling resistances of the belt on idlers. Motion resistances, including rolling resistances, have long remained an object of research. Extensive laboratory tests and increasingly detailed theoretical models allow a better insight into the phenomena involved in the movement of the belt on the conveyor. Different research results place different weight on the influence of individual parameters (such as unit load or phase lag angle) on belt rolling resistance. The greatest inconsistencies, however, are observed in the analyses of belt speed impact, which is occasionally not even included in some models. This paper discusses the known and commonly used methods for determining the rolling resistances of the belt on idlers. It also proposes a new solution, which employs the analysis of strain variation in the belt for successive load cycles to determine whether belt speed has a direct influence on the value of rolling resistance.
\end{abstract}

\section{Introduction}

Belt conveyors are an indispensable means of transport in a number of industries, but their most significant application is in the mining industry. Modern conveyors prove highly reliable and capable of transporting material over distances of several dozen kilometers, and with efficiencies of up to several tens of thousands of tones per hour. Therefore, the main goal for further improvements in the field is to maintain the transportation capabilities of belt conveyors while reducing their energy consumption [1]. Limiting power demand of belt conveyors directly and significantly limits the costs related to the operation of a mining transport system. This task may be achieved by first identifying what factors or operating parameters mostly affect the energy consumption levels. The energy consumption of belt conveyors results from motion resistances and therefore only a detailed analysis of their components may indicate where greatest reductions are possible. The issue of reducing motion resistances has long been the object of study in many research centers worldwide [2-5]. The influence of such parameters as unit belt load or operating temperature on individual components of motion resistances remains beyond doubt. Belt speed, however, either is given various degrees of importance in theoretical models or is not represented at all [6-9]. Unlike in-situ tests or even laboratory tests, theoretical analysis of strain in conveyor belt may provide definite answers on whether belt speed directly affects belt rolling resistances.

\section{Symbols}

$\sigma \quad$ - compressive stresses acting on the belt, $[\mathrm{Pa}]$

$\xi \quad$ - measure of belt damping, [-]

$t_{0} \quad$ - duration of a single load cycle, $[\mathrm{s}]$

$t_{m} \quad$ - time to maximum transverse strain of the belt, [s]

$\Delta t \quad$ - belt transit time between idler sets, [s]

$\omega_{0} \quad$ - angular velocity of the idler, $\left[\frac{\mathrm{rad}}{\mathrm{s}}\right]$

$v_{t} \quad$ - belt speed, $\left[\frac{m}{s}\right]$

$\delta \quad$ - phase lag angle, $[\mathrm{rad}]$

$\varepsilon \quad$ - transverse strains, [-]

$\varepsilon_{1} \quad$ - particular solution (viscous flow), [-]

$\varepsilon_{2} \quad$ - general solution (typical for harmonic load), [-]

$\tau_{0} \quad$ - time constant of the belt's model, [s]

$D_{k} \quad$ - idler diameter, [m]

$l_{k} \quad$ - idler set spacing, [m]

$E_{c} \quad$ - modulus of elasticity / resultant modulus of the belt under transverse compression (allowing for a number of plies in the belt), $[\mathrm{Pa}]$

\footnotetext{
* Corresponding author: martyna.konieczna@pwr.edu.pl
} 


\section{Motion resistances and energy- consumption of belt conveyors}

During the operation of a belt conveyor, a number of physical phenomena occur, which lead to energy conversions. Each case of energy loss is caused by another component of motion resistances. These resistances include three groups: main resistances, concentrated resistances and lift resistances. Lift resistances occur in the inclined sections of the conveyor flight and are attributed to the lifting of both the belt and the transported material. Concentrated resistances include the resistances on the head pulley, the drive pulley, the tail pulley, the take-up pulley and on the impact idlers. Main resistances accompany belt movement along the complete flight of the belt conveyor. They include idler rotational resistances, belt flexure resistances, flexure resistances of bulk material, belt slip resistances and rolling resistances of belt on idlers. Main resistances are the dominant component of motion resistances for conveyors having lengths greater than $80 \mathrm{~m}$. In turn, up to $60 \%$ of main resistances may be attributed to belt rolling resistances. Consequently, these resistances may decisively affect the values of motion resistances, and therefore research into energy-efficient belt conveyor solutions should first focus on a detailed analysis of factors which may influence belt rolling resistances. Belt rolling resistance (also referred to as belt indentation resistance) is the result of a cyclical indentation action of the idler into the pulley cover. Due to viscoelastic properties of the belt, its rolling resistance depends on a number of components, such as ambient temperature, load, or the geometry of the system.

\section{Calculation models}

The known and commonly used models for calculating belt rolling resistances can be classified into two groups $[10,11]$. The first group includes the Finite Element Method (FEM) proposed by Wheeler [9], and the Boundary Element Method (BEM) proposed by Qui [12]. Both methods were verified against either test results or the results independently obtained by other researchers. These models are based on the geometry of the system, which is divided into small sections, and the results are subsequently approximated for the whole system. The second group comprises theoretical models. Rolling resistances are calculated in these models in relation to a number of various factors. Authors of these methods use structural parameters of the conveyor and the load of the system. Authors such as May [13] and Hunter [6] also relate rolling resistance to belt speed. Jonkers, Spaans or Lodewijks [7, 8, 14], on the other hand, use phase lag angle and do not establish a direct link between the eventual rolling resistance values and belt speed.

\section{Theoretical analysis}

The significant influence that belt movement speed has on motion resistances in the conveyor is beyond doubt. Belt speed directly affects both the resistances which occur at the material feeding point (concentrated resistances) and some components of main resistances. In order to unequivocally determine the relationship between belt rolling resistances and belt speed, studies must focus on the physical phenomena leading to energy conversions. Information on this process is obtained from the hysteresis loop. It is a diagram representing the relationship between stress and strain in time. A belt conveyor operated in actual conditions experiences harmonic loads when the belt passes the idler support. In the first loading cycle, the hysteresis loop starts from zero stress and strain (Fig. 1). Belt loads are accompanied by temporary strain. The viscoelastic properties of the belt cause this strain to occur beyond the contact zone between the belt cover and the idler coat. The belt resumes its initial state with some delay (phenomenon known as viscous flow). The spaces between idler sets on the conveyor are sufficiently wide for the belt to resume its initial state. In such case, each loading cycle may be considered as the first cycle. In order to verify this fact, an analysis should be performed whether a relationship exists between the final strain and the initial strain in successive loading cycles.

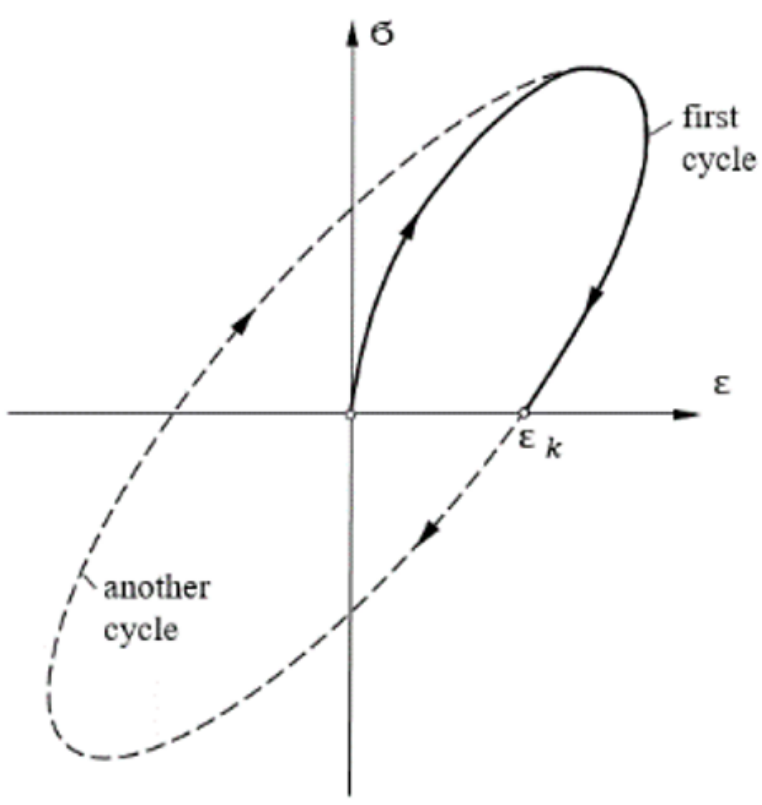

Fig. 1. Hysteresis loop for the first and for the successive loading cycles. 
II cycle III cycle

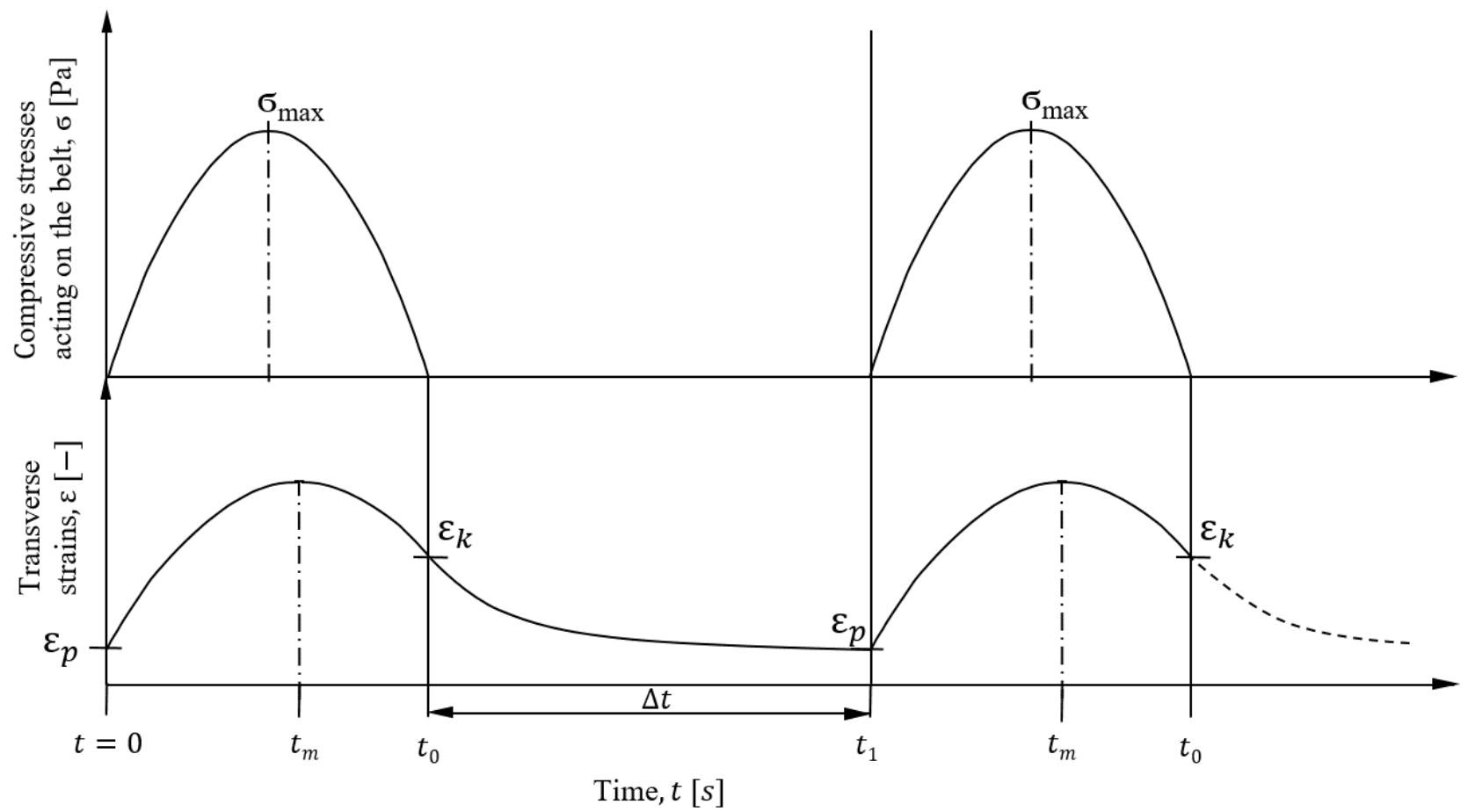

Fig. 2. Diagram of stress and strain in the belt on successive idler supports.

If an initial strain is assumed to already exist in each successive loading cycle (except for the first cycle), investigations must demonstrate whether the belt, after being subjected to the final strain in a cycle, will have sufficient time to resume its original shape while traveling between the idler sets. Compressive stresses acting on the belt occur in its contact zone.

Compressive stresses are a simple harmonic function, which may be expressed with the following equation:

$$
\sigma=\sigma_{0} \cdot \sin \left(\omega_{0} \cdot t\right)
$$
form:

On the belt conveyor, this function takes the following

$$
\sigma=E_{c} \cdot\left(\varepsilon+\tau_{0} \cdot \frac{d \varepsilon}{d t}\right)
$$

Such stress distribution is accompanied by transverse strain, which has two components. The particular solution includes viscous belt flow due to successive loading cycles on the idler supports as well as the spaces between idler sets. The general solution, on the other hand, is a typical function of harmonic loads which allows for the delay between the stress and the strain (phase lag $\delta$ ).

For the first cycle:

$$
\varepsilon_{1 m}=\frac{\sigma_{0}}{2 \cdot E_{C}} \cdot \sin (2 \delta) \cdot e^{-\frac{t_{m}}{\tau_{0}}}
$$

with the idler and if the load on the system is assumed to be constant, $\sigma_{\max }$ is identical for each cycle. Maximum strain occurs in the location where the belt passes over the central part of the idler. Therefore, $\varepsilon_{\max }$ is shifted in relation to $\sigma_{\max }$.

$$
\begin{aligned}
& \varepsilon_{2 m}=\frac{\sigma_{0}}{E_{C}} \cdot \cos \delta \cdot \sin \left(\omega_{o} \cdot t_{m}-\delta\right) \cdot e^{-\frac{t_{m}}{\tau_{0}}} \\
& =\frac{\sigma_{0}}{E_{C}} \cdot \cos \delta
\end{aligned}
$$

While belt strain at the end of the first cycle is:

$$
\begin{gathered}
\varepsilon_{1 k}=\frac{\sigma_{0}}{2 E_{C}} \cdot \sin (2 \delta) \cdot e^{-\frac{t_{0}}{\tau_{0}}} \\
\varepsilon_{2 k}=\frac{\sigma_{0}}{E_{C}} \cdot \cos \delta \cdot \sin \left(\omega_{o} \cdot t_{0}-\delta\right) \cdot e^{-\frac{t_{0}}{\tau_{0}}} \\
=-\frac{\sigma_{0}}{E_{C}} \cdot \cos \delta \cdot \sin \delta \\
=\frac{\sigma_{0}}{2 E_{C}} \cdot \sin (2 \delta) \cdot e^{-\frac{t_{0}}{\tau_{0}}}-\frac{\sigma_{0}}{E_{C}} \cdot \cos \delta \cdot \sin \delta \\
\varepsilon_{k}=\frac{\sigma_{0}}{E_{C}} \cdot \frac{\sin (2 \delta)}{2} \cdot\left(e^{-\frac{t_{0}}{\tau_{0}}}-1\right)
\end{gathered}
$$

Therefore, $\varepsilon_{p}$ should be assumed to correspond to the strain which remains in the belt after it passes between idler supports. 


$$
\varepsilon(\Delta t)=\varepsilon_{p}=\varepsilon_{k} \cdot e^{-\frac{\Delta t}{\tau_{0}}}
$$

As can be seen, the relationship between $\varepsilon_{p}$ and $\varepsilon_{k}$ depends on the time constant in the belt model. This observation leads to the following thesis:

$$
f\left(v_{t}\right)=-\frac{\Delta t}{\tau_{0}}
$$

When analyzing the strain in the second cycle:

$$
\varepsilon_{p}=\frac{\sigma_{0}}{E_{C}} \cdot \frac{\sin (2 \delta)}{2} \cdot\left(e^{-\frac{t_{0}}{\tau_{0}}}-1\right) \cdot e^{-\frac{\Delta t}{\tau_{0}}}
$$

Based on an assumption that no stress relief occurs in the belt, in the contact zone with the idler:

$$
\begin{gathered}
\varepsilon_{\max }=\varepsilon_{1 m}+\varepsilon_{2 m}+\varepsilon_{p} \\
\varepsilon_{\max }=\frac{\sigma_{0}}{2 E_{C}} \cdot\left(\sin 2 \delta \cdot e^{-\frac{t_{m}}{\tau_{0}}}+\frac{\sigma_{0}}{E_{C}} \cos \delta+\sin 2 \delta \cdot e^{-\frac{\Delta t}{\tau_{0}}} \cdot\left(e^{-\frac{t_{0}}{\tau_{0}}}-1\right)\right)
\end{gathered}
$$

In order to simplify the equation, the phase lag angle $\delta$ may be substituted with the measure of damping $\xi$.

$$
\begin{gathered}
\delta=\frac{\pi}{2} \cdot \frac{1-\xi}{1+\xi} \\
\varepsilon_{p}(\xi)=\frac{\sigma_{0}}{2 E_{C}} \cdot\left(\sin \left(\pi \cdot \frac{1-\xi}{1+\xi}\right) \cdot e^{-\frac{\Delta t}{\tau_{0}}}\left(e^{-\frac{t_{0}}{\tau_{0}}}-1\right)\right)
\end{gathered}
$$

Thus, for the second and each successive cycle in the function $\xi$, the equation describing initial strain takes the following form:

$$
\varepsilon_{p}=\frac{\sigma_{0}}{2 E_{C}} \cdot\left(\sin \left(\pi \cdot \frac{1-\xi}{1+\xi}\right) \cdot e^{-\frac{\Delta t}{\tau_{0}}}\left(e^{-\frac{\pi}{\operatorname{tg}\left(\frac{\left.\pi \cdot \frac{1-\xi}{2} \cdot \frac{1}{1+\xi}\right)}{1}\right.}}-1\right)\right)
$$

The function is still dependent on $e^{-\frac{\Delta t}{\tau_{0}}}$. Therefore, it must be verified whether the power exponent depends in this case directly on speed (whether $-\frac{\Delta t}{\tau_{0}}$ is a function depending on $v_{t}$ ).

The relationship between the time constant of the belt model and the phase lag angle is described with the following equation:

$$
\omega_{0} \cdot \tau_{0}=\operatorname{tg} \delta
$$

Angular speed, on the other hand, may be determined as follows:

$$
\omega_{0}=\frac{2 v_{t}}{D_{k}}
$$

where:

$$
v_{t}=\frac{l_{k}}{\Delta t}
$$

Thus:

$$
-\frac{\Delta t}{\tau_{0}}=-\frac{\Delta t \cdot \omega_{o}}{\operatorname{tg} \delta}=-\frac{2 l_{k}}{\operatorname{tg} \delta \cdot D_{k}}
$$

Therefore, the dependence (9) is influenced not only by the structural parameters of the conveyor but also by the phase lag angle.

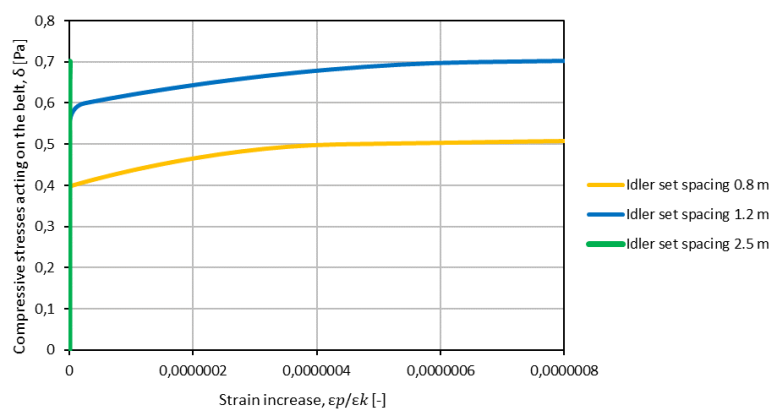

Fig. 3. Relationship between phase lag angle and strain growth.

The diagram in Fig. 3 was plotted with idler set spacing assumed to be at $0.8 \mathrm{~m}, 1.2 \mathrm{~m}$ and $2.5 \mathrm{~m}$ in the top run and the idler diameter to be $0.2 \mathrm{~m}$. The variants designated in green and in yellow correspond to extreme distances between the idler sets. The blue line indicates the $1.2 \mathrm{~m}$ spacing, which is the most typical distance in practical applications. Thus, in the case of a typical belt conveyor, a potential strain growth in the belt in successive cycles may be due to the high value of phase lag angle.

\section{Conclusion}

The theoretical analysis here presented indicates that speed does not have a direct impact on the rolling resistance value of the belt on idlers. The influence of phase lag angle on belt rolling resistance is commonly known. Typically, the values of $\delta$ do not exceed 0.4 radian. In the case of greater phase lag angles, on the order of $0.5-0.6$ radian, rolling resistances become also influenced by idler set spacing. In such case, the ratio of the initial strain to the final strain of the previous cycle is between $1 \cdot 10^{-7}$ and a maximum of $8 \cdot 10^{-7}$. This increment is still negligible. Importantly, the energy-efficient belts, which are currently becoming increasingly popular, have phase lag angles smaller than traditional belts. In the case of such belts, strain growth will not be observed even if idler sets are spaced at $0.8 \mathrm{~m}$.

Financial support by the project: Methods for assessing the energy efficiency of transport systems No. 0401/0131/17. 


\section{References}

1. L. Gładysiewicz and M. Konieczna, Mining Science, 23, 105-120 (2016)

2. R. Król, W. Kisielewski, D. Kaszuba, L. Gładysiewicz, International Journal of Mining, Reclamation and Environment 31(1), 78-90 (2017)

3. M. Bajda and R. Król, Procedia: Earth and Planetary Science, World Multidisciplinary Earth Sciences Symposium WMESS, Vol. 15, 702-711 (2015)

4. D. Woźniak, L. Gładysiewicz, M. Konieczna, E3S Web Conf. 29 (2018)

5. C. Wheeler and P. Munzenberger, International Materials Handling Conference (Beltcon 16) (2011)

6. S.C. Hunter, Journal of Applied Mechanics, 34, 612617 (1961)

7. C.O. Jonkers, The indentation rolling resistance of belt conveyor - a theoretical approach, Fordern und Heben (1980)

8. G. Lodewijks, Bulk Solids Handling, 15, 1, 15-22 (1995)

9. C. Wheeler, Analysis of the main resistance of belt conveyors. $\mathrm{PhD}$ thesis, University of Newcastle, Australia (2003)

10. S. Drenkelford, Energy-saving potential of Aramidbased conveyor belts, Delft University of Technology (2015)

11. T.J. Rudolphi and A.V. Reicks, Rubber Chemistry and Technology, 79, 307-319 (2006)

12. X. QIU, Journal of Engineering Mechanics, 132, 11, 1241-1251 (2006)

13. W.D. May, E.L Morris., D. Atack, Journal of Applied Physics, 30, 1713-1724 (1959)

14. C. Spaans, Bulk Solids Handling, 11, 4, 809-826 (1991) 